\title{
Rutura Espontânea de Adrenocarcinoma em Portador de Hiperplasia Adrenal Congênita
}

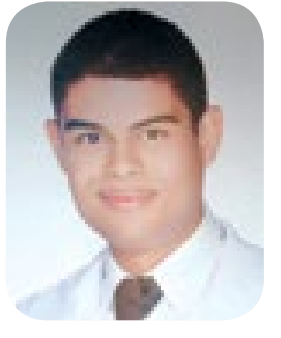

\author{
Rogger Gonçalves Ribeiro ${ }^{1}$, Alina Coutinho Rodrigues Feitosa ${ }^{1}$ \\ Palavras-chave: hiperplasia suprarrenal congênita, esteroide 21-hidroxilase, \\ neoplasia das glândulas suprarrenais
}

Key words: congenital adrenal hyperplasia, steroid 21-hydroxylase, adrenal gland neoplasms

\section{INTRODUÇÃO}

A hiperplasia adrenal congênita (HAC) é um grupo de doenças de herança autossômica recessiva, resultante da deficiência de enzimas necessárias à síntese dos esteroides adrenais. A mais frequente é a deficiência da 21-hidroxilase, responsável por mais de $90 \%$ dos casos $^{1}$. A expressão clínica é relacionada ao grau de deficiência da atividade da 21 hidroxilase e varia da forma não clássica, leve, assintomática ou com sinais de hiperandrogenismo pós-natal à forma clássica, com graves manifestações de virilização ao nascimento, desidratação e choque hipovolêmico. A síntese deficiente de cortisol na adrenal estimula a produção de ACTH por mecanismo de alça de retroalimentação e resulta em acúmulo de precursores do cortisol, que são desviados para a biossíntese de hormônios sexuais, sendo responsáveis pela virilização. A deficiência concomitante da aldosterona resulta em distúrbios eletrolíticos e desidratação grave. Cerca de $75 \%$ dos casos da forma clássica são perdedores de sal e o restante, a forma virilizante simples. No Brasil, a incidência da forma clássica é de 1:10.000 a 20.000 nascidos vivos e o diagnóstico é feito por meio da avaliação do perfil hormonal adrenocortical e genótipo, em casos selecionados ${ }^{2}$.

A estimulação cortical adrenal crônica por excesso de ACTH em pacientes com HAC aumenta o risco de tumorigênese adrenal ${ }^{3}$. Portadores de HAC apresentam, mais frequentemente, tumores adrenais que a população geral. Os tipos histológicos de tumor adrenal incluem os adenomas, mielolipomas, hemangiomas ${ }^{4}$, tumores virilizantes e feminilizantes e, muito raramente, carcinomas adrenocorticais (CAC).

A incidência de CAC em portadores de HAC é, no entanto, desconhecida e o quadro clínico tem apresentação variável, desde tumores não-funcionantes com síndromes compressivas a formas feminilizantes, cushingoides e virilizantes.
Relatamos o caso de um paciente adulto, cariótipo $X X$, portador de hiperplasia adrenal congênita não tratada, com virilização completa e identificação sexual masculina que se apresentou com choque hemodinâmico por abdômen agudo hemorrágico, devido à rutura espontânea de tumor adrenal que revelou ser CAC.

\section{RELATO DE CASO}

C.A.P.N., sexo masculino, casado, 28 anos, foi encaminhado ao serviço de Endocrinologia e Metabologia em setembro de 2016 para avaliação pré-operatória de adrenalectomia à direita, devido à tumoração. Havia sido submetido, em novembro de 2015, à laparotomia exploradora por abdome agudo hemorrágico, detectado após quadro súbito de dor abdominal lancinante com choque hemodinâmico, sendo identificada rutura espontânea de tumoração em adrenal esquerda, com volumoso hemoperitônio. O diagnóstico anátomopatológico foi de adrenocarcinoma adrenal à esquerda. Permaneceu em seguimento oncológico e, em maio de 2016, ressonância de abdômen revelou tumoração em adrenal remanescente: formação nodular, de contornos regulares, com isossinal em T1 e hipossinal em T2, com realce ao meio do contraste, planos de clivagem com as estruturas adjacentes, localizada em suprarrenal direita, medindo 5,1x3,7 cm. Foi indicada adrenalectomia.

Paciente é filho de pais consanguíneos (primos em primeiro grau), referiu aparecimento de pêlos em áreas andrógeno-dependentes, com aproximadamente 5 anos de idade, aceleração do desenvolvimento estatural e muscular e parada do crescimento por volta dos 13 anos (altura final de $134 \mathrm{~cm}$ ), quando foi encaminhado a um serviço de genética. Nesta unidade foi detectada a ausência de gônadas na bolsa escrotal, foi identificado genitália interna feminina e submetido à histerectomia e foi orientado a usar uma medicação que não lembrava o nome. Não fez uso do remédio e abandonou o tratamento até a idade de 28 anos. 
Ao exame físico apresentava fenótipo masculino, baixa estatura e expressiva constituição muscular em membros. Altura de $134 \mathrm{~cm}$, peso de $46 \mathrm{~kg}$. Apresentava barba e pelos terminais em torax anterior, costas, pescoço, pernas e abdomen. O exame pélvico revelava aspecto masculino da genitália, pilificação Tanner $V$, falus de $12 \mathrm{~cm}$, bolsa escrolal fusionada, escurecida, enrugada e hipoplásica com ausencia de gônadas palpáveis. A pressão arterial era de 170×130 mmHg e quência cardíaca de 76 bpm.

A avaliação laboratorial revelou hiperandrogenismo com supressão de gonadotrofinas, elevação do ACTH e da 17 hidroxiprogesterona séricos e hipocortisolismo. Hemoglobina em 12,2 g/dl, hematócrito em $46 \%$, leucócitos em 7840, com diferencial normal, plaquetas em 280.000; sódio em $135 \mathrm{mEq} / \mathrm{L} ; \mathrm{K}$ 4,39 mEq/L; FSH inferior a 0,3 mUl/mL; testosterona total: $988,7 \mathrm{ng} / \mathrm{dL}$ (valor de referência [VR] adultos: 241-827; mulheres: 14-76); dehidroepiandrosterona: $18,5 \mathrm{ng} / \mathrm{mL}$ (VR Feminino: 1-12; Masculino: 3-11), sulfato de dehidroepiandrosterona: $827 \mathrm{ug} / \mathrm{dL}$ (VR 80-550); androstenediona: superior a $10 \mathrm{ng} / \mathrm{mL}$ (VR Homens: 0,5-4,8); 17 alfa-hidroxi-progesterona 1039,8 $\mathrm{ng} / \mathrm{dL}$ (VR Feminino: fase folicular: 10-80; fase lútea: 60-230; pós-menopausa:13-51; apos estimulo com ACTH: ate 1000) progesterona: 10,91 $\mathrm{ng} / \mathrm{mL}$ (VR fase folicular: 3,34 25,56; pós-menopausa: até 0,73 ; masculino adulto: 0,28-1,22); ACTH: $135 \mathrm{pg} / \mathrm{mL}$ (VR 7,2-63,3); cortisol das 08h: 5,3 $\mu \mathrm{g} / \mathrm{dL}$ (VR 6,25-18,52); TSH 2,088 uUl/mL (VR 0,34-5,6); T4L 0,9 ng/dL (VR 0,7-1,8).

Foi submetido à laparotomia exploradora, que mostrou tumoração em adrenal com maior diâmetro em 10 $\mathrm{cm}$, sendo feita a ressecção completa. Não havia linfadenomegalias, sinais de infiltração tumoral adjacente ou evidência macroscópica de carcinomatose. No intraoperatorio foi feito uso de hidrocortisona em bolus de 100mg e mantida a dose venosa de $100 \mathrm{mg}$ a cada 08/08h, com redução de 50\% a cada $24 \mathrm{~h}$ até transição para prednisona, na dose de $5 \mathrm{mg}$ associada a fludrocortisona, $100 \mathrm{mcg}$ pela manhã. Foi prescrito, também, cipionato de testosterona, $200 \mathrm{mg}$, por via intramuscular a cada 14 dias e com alta hospitalar normotenso sem hipotensaao ortostatica e com orientações sobre sinais e sintomas de insuficiência adrenal aguda. $O$ diagnóstico anátomopatológico foi de adenoma adrenocortical à direita.

\section{DISCUSSÃO}

umores adrenocorticais (TACs) são, em geral, unilaterais e classificados em adenomas adrenocorticais benignos ou CAC. Em portadores de HAC, a prevalên- cia de massas adrenais é elevada, chegando a $82 \%$, em homozigotos, e $45 \%$, em heterozigotos ${ }^{5}$ e estes números podem ser ainda mais elevados em pacientes com tratamento inadequado ou não tratados ${ }^{6}$. Carcinomas adrenocorticais são raríssimos. No referido caso, o paciente apresentou CAC a esquerda com rutura espontânea e formação de hemoperitônio e adenom drenal à direita.

A prevalência estimada de CAC na população gera é de 1 a 2 casos por milhão/ano. Fato de destaque internacional é a elevada incidência de CAC em crianças no Sudeste brasileiro. São 2,9 a 4,2 casos por mihão/ano, comparados à eslinada mundial de 0,2 a 0,3 caso/milhão/ano, que é atribuída à presença elevada de mutação no gene supressor tumoral P537. Informaçoes sobre CAC em portadores de HAC são escassas, maioria por relatos de caso, sendo a incidência e prevalência desconhecidas.

A maioria dos portadores de CAC apresenta-se com sinais e sintomas de excessos hormonais. Um terço em sintomas de dor abdominal, plenitude e saciedade os terços restantes são incidentalmente identificado por meio de exames de imagem 8 . A apresentação clínica do CAC com rutura espontânea é raríssima, sendo 0 abdômen agudo, mais raro ainda. Nos casos relatados, os CAC eram, em maioria, não-funcionantes ${ }^{9}$ apenas um foi funcionante ${ }^{10}$. O presente caso é extremamente inusitado, pois é o primeiro relato, ao nosso conhecimento, de rutura espontânea de CAC funcionante em portador de HAC em que a virilização estava presente desde o nascimento e o estímulo trófico cronico do ACTH seria uma das etiologias prováveis. No referido caso, não era conhecida pela equipe cirúrgica a história prévia de HAC e, na abordagem cirurgica emergencial, não foi identificada massa em adrenal remanescente. $O$ seguimento após 06 meses revelou imagem contralateral sugestiva de tumor e necessidade de nova abordagem. Para acompanhamento da insuficiència adrenal crônica que se sucederia apos a adrenalectomia foi encaminhado pa serviço de endocrinologia, momento em que foi feito estabelecido o diagnostico de HAC.

Os desafios, ao momento do diagnóstico da HAC, oram: 1. não era possivel definir se a massa adrena direita era benigna ou maligna sem a ressecção completa da adrenal; 2. a ressecção da adrenal remanescente resultaria em insuficiência adrenal crônica com necessidade de reposição com corticoide e mineralocorticoide pelo resto da vida; 3 . a ressecção da adren genos que levou à virilização e, em sendo a identifi- cação sexual do paciente masculina, seria necessário por testosterona exógena cronicamente.

Considerando-se a HAC não-tratada, era possíve que lesão na adrenal remanescente fosse benigna, devido ao estímulo crônico do ACTH, promovendo hiperplasia/adenoma. Entretanto, não foi possível definir malignidade por imagem e a detecção prévia de CAC aumentava a suspeita de tumoração maligna. A impossibilidade de diagnostico definitivo sem a ressecção completa da lesão adrenal não permitiu a observação exclusiva associada à reposição hormonal. A confirmação da benignidade antes da retirada da adrenal teria sido importante, pois a abordagem de massas adrenais em pacientes com HAC inadequadamente tratados ou não tratados pode ser feita apenas com a reposição hormonal e acompanhamento da imagem. Ao retira a adrenal remanescente, o paciente tornar-se-ia um portador de insuficiência adrenal crônica, com necessidade de reposição de corticoide e mineralocorticoide o resto da vida e, adicionalmente, seria extinta a fonte produtora dos andrógenos, a qual mantinha o fenótipo masculino. O paciente, genotipicamente feminino (cariótipo $\mathrm{XX}$ ), retomaria sua produção de hormônios sexuais femininos, pois os ovarios estavam presentes. Sendo assim, foi feila a reposiçáo com testosterona para manter as características sexuais masculinas.

\section{CONCLUSÃO}

Relatamos o caso de um paciente adulto, portado de hiperplasia adrenal congenita não tratada, que cursou com choque hemodinâmico por abdômen agudo hemorrágico, devido à rutura espontânea de $\mathrm{CAC}$. Esta intercorrencia e extremamente rara em CAC e nunca foi descrita em portador de HAC.

\section{REFERÊNCIAS}

1.White PC, Speiser PW. Congenital adrenal hyperplasia due to 21-hydroxylase deficiency. Endocr Rev 2000; 21:245.

2. Speiser PW, Azziz R, Baskin LS, Ghizzoni L, Hensle TW, Merke DP, et al. Congenital adrenal hyperplasia due to steroid 21-hydroxylase deficiency: an Endocrine Society clinical practice guideline. J Clin Endocrinol Metab. 2010;95(9):4133-60.

3. Barzon L, Maffei P, Sonino N, Pilon C, Baldazzi L, Balsamo $A$. The role of 21-hydroxylase in the pathogenesis of adrenal masses: Review of the literature and focus on our own experience. J. Endocrinol. Invest. 30 615-623, 2007

4. Pignatelli D, Vendeira P, Cabral AC: Adrenal in cidentalomas: adrenal hemangioma in a patient with congenital adrenal hyperplasia South Med J, 1998 91(8):775-79.

5. $\mathrm{S}$ Jaresch et al. Adrenal Incidentaloma and $\mathrm{Pa}$ tients With Homozygous or Heterozygous Congenit Adrenal Hyperplasia. J Clin Endocrinol Metab 74 (3) 685-689. 3 1992.

6. Wang J, Bissada MA, Williamson $\mathrm{HO}$, Yakout $\mathrm{H}$ Bissada NK.Adrenal tumors associated with inadequaely treated congenital adrenal hyperplasia. Can J Uro (2002 Jun;9(3):1563-4.

7. Pinto EM, Billerbeck AE, Villares MC, Domenice $S$, Mendonça BB, Latronico AC. Founder effect for the highly prevalent R337H mutation of tumor suppresso p53 in Brazllian patients with adrenocortical tumors. Arq Bras Endocrinol Metabol. 2004 Oct;48(5):647-50. pub 2005 Mar 7

8. Else T, Kim AC, Sabolch A, Raymond VM, Kandathil A, Caoili EM, Jolly S, Miller BS, Giordano TJ Hammer GD. Adrenocortical carcinon Reviews, April 2014, 35(2):282-326.

9. Symeonidis D, Chatzinikolaou I, Koukoulis G Mamaloudis I, Tepetes K. Adrenocortical carcinoma presenting with signs of acute abdomen. Case Rep Surg. 2013;2013:132726. Epub 2013 Feb 3 .

10. Chung JO, Cho DH, Lee JH, Kwon DD, Chung DJ, Chung MY. Spontaneous Rupture of a Functioning Adrenocortical Carcinoma. Yonsei Med J. 2010 No 51(6):974-977.

1- Serviço de Clínica Médica do Hospital Santa Izabe

Endereço para correspondência:

roggergoncalves@yahoo.com.br 